\title{
Viscoelastic Properties of Concentrated Solutions of Randomly Branched Polystyrenes
}

\author{
Toshiro Masuda, Yoshiteru Nakagawa, * Yasuhiko Ohta, \\ and Shigeharu ONOGI \\ Department of Polymer Chemistry, Kyoto University, Kyoto, Japan.
}

(Received August 2, 1971)

\begin{abstract}
The viscoelastic properties of concentrated solutions of linear and randomly branched polystyrenes were measured and compared with each other. From the frequency dependence of the storage shear modulus $G^{\prime}$ and the loss modulus $G^{\prime \prime}$, the characteristic parameters in the terminal zone, such as zero-shear viscosity $\eta_{0}$ and steady-state compliance $J_{e}^{0}$, were evaluated. The dependence of these parameters on the molecular weight and concentration were discussed. The zero-shear viscosity and its dependence on molecular weight for the solution of randomly branched polystyrene are lower than those of linear polystyrene. The steady-state compliance is proportional to $M_{w^{0}}{ }^{.84}$ for the branched samples, while it does not depend on molecular weight for the linear polymer. These results lead to the suggestion that the maximum relaxation time is proportional to $M_{w^{2.3}}$ and $M_{w^{3.5}}$ for branched and linear polystyrene solutions, respectively. The concentration dependence of the steady-state compliance for the branched polymers is also much less than that of the linear ones. Comparing the frequency dependence curves of $G^{\prime}$ for the randomly branched and linear polystyrenes, there seems to be a significant difference in the shape of the relaxation spectra in the region associated with entanglement coupling. This behavior is considered to be due to the difference in the two chain structures.

KEY WORDS Viscoelasticity / Polystyrene / Branched Polymer / Viscosity / Steady-State Compliance / Molecular Weight / Concentration Dependence /
\end{abstract}

The effect of branching on the viscoelastic properties of concentrated systems of high polymers, ${ }^{1-10}$ such as concentrated solutions and melts, is one of the most important subjects of polymer science, both in the academic and industrial sense.

Recently, it has become possible to prepare branched polymers having a well-controlled structure and narrow distribution of molecular weights, and a few measurements of the rheological properties of such samples have been carried out. One of these studies is the measurement of stress relaxation for comb-shaped polystyrenes by Fujimoto, et al. ${ }^{11}$ and the other is our study of dynamic viscoelasticity for starshaped polystyrene. ${ }^{12}$ These studies for branched polymers having different types of branching show that zero-shear viscosity for the branched

\footnotetext{
* Present address: Sumitomo Chemical Co., 40, Tsukahara, Takatsuki, Osaka, Japan.
}

polymer is lower than that for the linear polymer so long as molecular weight is not too high and that the steady-state compliance for the former is about ten times higher than that for the latter. Though the relaxation modulus or storage modulus for the star branch polymer is about the same as that of the corresponding linear polymer in the rubbery plateau region, the comb-shaped polymer has a relaxation modulus lower than the corresponding linear polymer in that region.

In this paper, viscoelastic properties were measured for concentrated solutions of randomly branched polystyrene which has another type of branching. The experimental results were compared with those for concentrated solutions of linear polystyrenes.

\section{EXPERIMENTAL}

\section{Materials}

Randomly branched polystyrenes were prepared 
by the copolymerization of styrene monomer with a small amount of divinylbenzene (DVB). $\alpha, \alpha^{\prime}$-Azobisisobutyronitrile(AIBN) was used as the initiator. Conditions of copolymerization are shown in Table I. The copolymer of styrene

Table I. Copolymerization conditions for randomly branched samples

\begin{tabular}{cccccc}
\hline \multicolumn{2}{c}{ Molar ratio } & \multicolumn{2}{c}{$\begin{array}{c}\text { Polymeriza- } \\
\text { tion time, hr }\end{array}$} & $\begin{array}{c}\text { Con- } \\
\text { version }\end{array}$ & $\begin{array}{c}\text { Temp, } \\
\text { Styrene }\end{array}$ \\
\cline { 1 - 2 } 277 & 1 & 0.98 & 4 & 0.285 & 65 \\
\hline
\end{tabular}

and divinylbenzene used in this study was fractionated by the fractional precipitation method in a benzene-methanol system. The fractionation was repeated 2-6 times and ten fractions having the desirable molecular weight were selected as the samples. Linear polystyrenes used for the sake of comparison were prepared by bulk polymerization and were fractionated. by the same method mentioned above. The conversion of the polymerization was less than $20 \%$.

Weight- and number-average molecular weights and intrinsic viscosities at $\theta$-temperature for the samples employed in this study are tabulated in Table II. RB series and PS series in this

Table II. Operating conditions of GPC

\begin{tabular}{ccccc}
\hline Columns Solvent & $\begin{array}{c}\text { Temp } \\
{ }^{\circ} \mathrm{C}\end{array}$ & $\begin{array}{c}\text { Sample } \\
\text { concn, Injection } \\
\mathrm{g} / \mathrm{d} l\end{array}$ & $\begin{array}{c}\text { Flow } \\
\text { time, sec } \\
\mathrm{ml} / \mathrm{min}\end{array}$ \\
\hline $4 \times 3 \mathrm{ft}\left(\begin{array}{c}10^{4} \\
10^{5} \\
10^{6}\end{array}\right)$ THF & $45^{\circ} \mathrm{C}$ & 0.25 & 60 & 1
\end{tabular}

table represent the fractions of polystyrenes which were prepared by the copolymerization of styrene and DVB and those of the linear ones, respectively. $M_{n}$ of the branched and linear polymers was obtained by the osmotic pressure technique using a high speed membrane osmometer, Model 502, of Mecrolab Inc. and $M_{w}$ was determined from light-scattering data in methyl ethyl ketone. The intrinsic viscosity $[\eta]$ of all the samples was measured with dilute solutions in cyclohexane at $34.5^{\circ} \mathrm{C}$.

50 -wt $\%$ solutions of all ten samples and 20- and $10-w t \%$ solutions of two samples were used for measurements of the viscoelastic properties. As the solvent, Kaneclor 500 (KC5) was used which is a partially chlorinated diphenyl produced by Kanegafuchi Chemical Industry Co. The required amount of polymer and solvent (KC5) was perfectly dissolved in dichloromethane by stirring, and then the dichloromethane was completely removed by evaporation from the mixture in a vacuum oven.

\section{Measurement of Viscoelasticity}

The measurements of the viscoelastic properties of the samples were carried out with the concentric cylinder-type rheometer described in previous paper. ${ }^{12}$ The frequency of the oscillations ranged from $4 \times 10^{-3}$ to $0.5 \mathrm{~Hz}$. The measuring temperature ranged from 10 to $150^{\circ} \mathrm{C}$. The viscoelastic functions such as the storage shear modulus $G^{\prime}$ and loss modulus $G^{\prime \prime}$ were calculated by the use of the general equation given by Markovitz. ${ }^{13}$ Such $\log G^{\prime} v s . \log \omega$ curves as well as the similar curves for the loss modulus $G^{\prime \prime}$ at various temperatures can always be superposed into respective master curves at $50^{\circ} \mathrm{C}$ by use of the time-temperature superposition principle. The shift factor $a_{\mathrm{T}}$ obtained can be expressed by the following WLF-type equation:

$$
\log a_{\mathrm{T}}=\frac{-9.3\left(T-T_{\mathrm{r}}\right)}{88.6+\left(T-T_{\mathrm{r}}\right)}, \quad T_{\mathrm{r}}=50^{\circ} \mathrm{C}
$$

There is no difference between $a_{\mathrm{T}}$ for the branched polymer and that for the linear one.

\section{RESULTS AND DISCUSSION}

\section{Characterization of the Randomly Branched Poly- styrenes}

In the copolymerization of styrene and DVB, the conversion $\beta$ can be written as

$$
\beta=\left(n_{1}+n_{2}\right) /\left(N_{1}+N_{2}\right) \cong n_{1} / N_{1}
$$

where $n$ and $N$ denote the number of monomers, converted into polymer and in initial mixtures, respectively. The subscripts 1 and 2 are used for styrene and DVB, respectively. On the other hand, the density of branch points in the polymer chain, $\rho_{\mathrm{b}}$ is given as ${ }^{14}$

$$
\begin{aligned}
& \rho_{\mathrm{b}}=\nu / n_{1}=-C[1+(1 / \beta) \ln (1-\beta)] \\
& C=\left(k_{12}^{\prime} / \gamma_{1} k_{12}\right)\left(N_{2} / N_{1}\right)
\end{aligned}
$$


where is the total number of branch points in the system, $k_{12}$ is the reactivity constant of the first DVB bond, $k_{12}^{\prime}$ is that of the second, and $\gamma_{1}$ is the monomer reactivity ratio. Assuming $k_{12}^{\prime}=k_{12}$, and using the values, $N_{1} / N_{2}=277$, $\beta=0.285$, and $\gamma_{1}=0.57$ which is the average value of the three isomers ${ }^{15}$ of DVB, eqs 3 and 4 give $\rho_{\mathrm{b}}$ value as

$$
\rho_{b}=0.00112
$$

Then the branching parameter $\lambda$ and the molecular weight between branch points $\boldsymbol{M}_{\mathrm{b}}$ defined by the following equation

$$
\lambda=1 / M_{\mathrm{b}}=\rho_{\mathrm{k}} / M_{0}
$$

can be calculated as

$$
\lambda=1.078 \times 10^{-5} \text { and } M_{\mathrm{b}}=9.28 \times 10^{4}
$$

where $M_{0}$ is the molecular weight of monomers. This $M_{\mathrm{b}}$ value shows a close agreement with the molecular weight $9 \times 10^{4}$ at the first peak in gel-permeation chromatogram as shown in Figure 1 and the estimated values from the molecular weight dependence of the rheological parameters as will be discussed later.

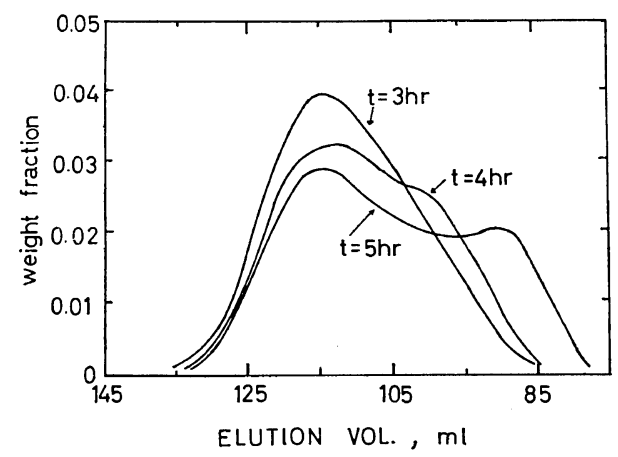

Figure 1. Gel-permeation chromatograms for the copolymer of styrene and divinylbenzene at previous polymerization times.

In Figure 1 are shown the gel-permeation chromatograms at various polymerization times for the copolymer of styrene and DVB in the preliminary experiments, which were performed under the same conditions as described in Table I. Each distribution curve in this figure was normalized for comparison purposes. The gelpermeation chromatograph(GPC) used in this study was a Model 200, manufactured by Waters Associates
Inc. and the operation conditions are shown in Table II. Although the linear and branched polymers of the same molecular weight give different elution volumes because of the difference in dimension of the polymer chain, the change in the molecular-weight distribution with polymerization times can be learned qualitatively from this figure. At shorter time $(t, 3 \mathrm{hr})$, the distribution curve has a single peak at the elution volume of $115 \mathrm{ml}$, corresponding to the molecular weight of a linear polymer of about $9 \times 10^{4}$. At longer times ( $t, 4$ and $5 \mathrm{hr}$ ), however, another peak appears at higher molecular weight (lower elution volume) and the molecular weight of this peak seems to be shifted to the higher molecular weight side, while the former peak remains at constant molecular weight. Therefore it is suggested that the peak at the molecular weight of about $9 \times 10^{4}$ corresponds to linear polymer and the peak at the higher molecular weight to be branched one which is produced by the addition of the polymer radical to the second vinyl group of divinylbenzene. According to eq 3 and $5, M_{\mathrm{b}}$ changes from $1.5 \times 10^{5}$ to $8 \times 10^{4}$ with increasing the polymerization time from $3 \mathrm{hr}$ to $5 \mathrm{hr}$. However, this small difference in $M_{\mathrm{b}}$ value does not appear in the GPC chart as shown in Figure 1.

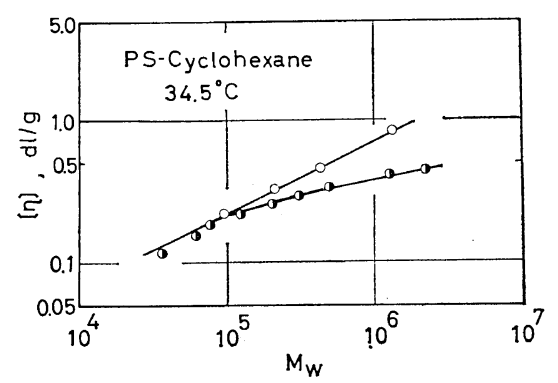

Figure 2. Intrinsic viscosity plotted against the weight-average molecular weight. The open circles and right black circles represent the data for the PS and RB series, respectively.

Figure 2 shows the double logarithmic plot of $[\gamma]$ against $M_{w}$, where the open circles and the right black ones represent the data for the linear and branched samples, respectively. [ $\eta]$ for the linear polystyrene is proportional to $M_{w}^{0.5}$. On the other hand, the values of $[\eta]$ 
for RB series fit well on the straight line for linear polymer at first, and deviate from the line, becoming proportional to $M_{w}^{0.2-0.25}$, with increasing molecular weight. It is suggested ${ }^{16}$ that the samples having lower $M_{w}$ (RB1-3) are linear polymers and the others (RB4-10) are randomly ones. Moreover, the molecular weight, $1.0 \times 10^{5}$, at which $[\eta]$ for $\mathrm{RB}$ series deviates from that linear polymer is almost the same as those evaluated from GPC and the kinetic theory of copolymerization, as discussed previously.

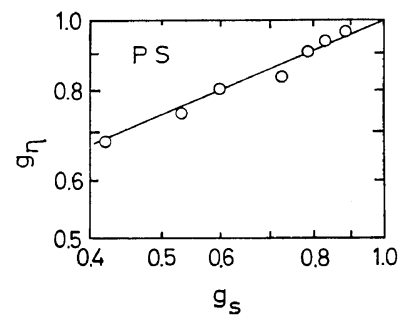

Figure 3. $g_{\eta}=\left([\eta]_{\mathrm{b}} /[\eta]_{1}\right)^{1 / 3}$ plotted against $g_{\mathrm{s}}=$ $\left(\left\langle s^{2}\right\rangle_{\mathrm{b}} /\left\langle s^{2}\right\rangle_{1}\right)^{1 / 2}$ for randomly branched polystyrenes.

Figure 3 shows the relation between $g_{\eta}=\left([\eta]_{\mathrm{b}} /\right.$ $\left.[\eta]_{1}\right)^{1 / 3}$ and $g_{\mathrm{s}}=\left(\left\langle s^{2}\right\rangle_{\mathrm{b}} /\left\langle s^{2}\right\rangle_{1}\right)^{1 / 2}$, doubly logarithmically plotted where the subscripts $b$ and 1 denote the branched and linear polymer, respectively, and $\left\langle s^{2}\right\rangle$ is the mean-square radius of gyration. Because the exact values of $\left\langle s^{2}\right\rangle$ could not obtained from light scattering, the

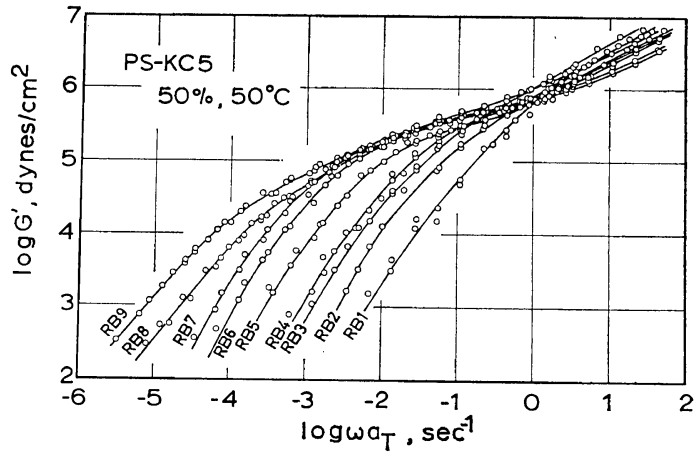

Figure 4. Master curves of $G^{\prime}$ for the 50-wt $\%$ solutions of $\mathrm{RB}$ series in $\mathrm{KC5}$. The reference temperature is $50^{\circ} \mathrm{C}$.

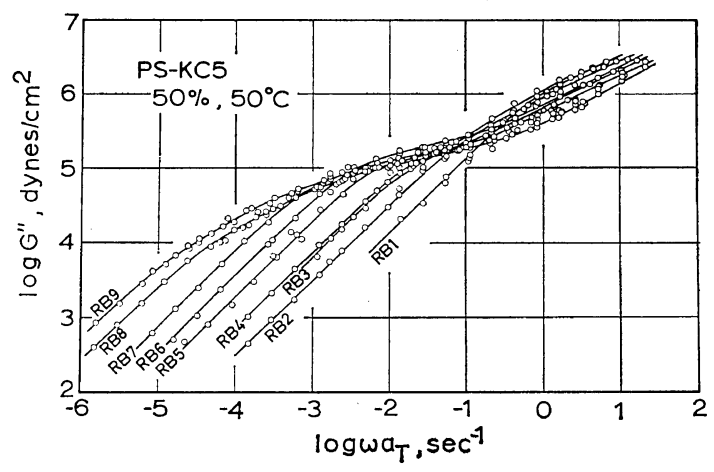

Figure 5. Master curves of $G^{\prime \prime}$ for the $50-\mathrm{wt} \%$ solutions of RB series in KC5. The reference temperature is $50^{\circ} \mathrm{C}$.

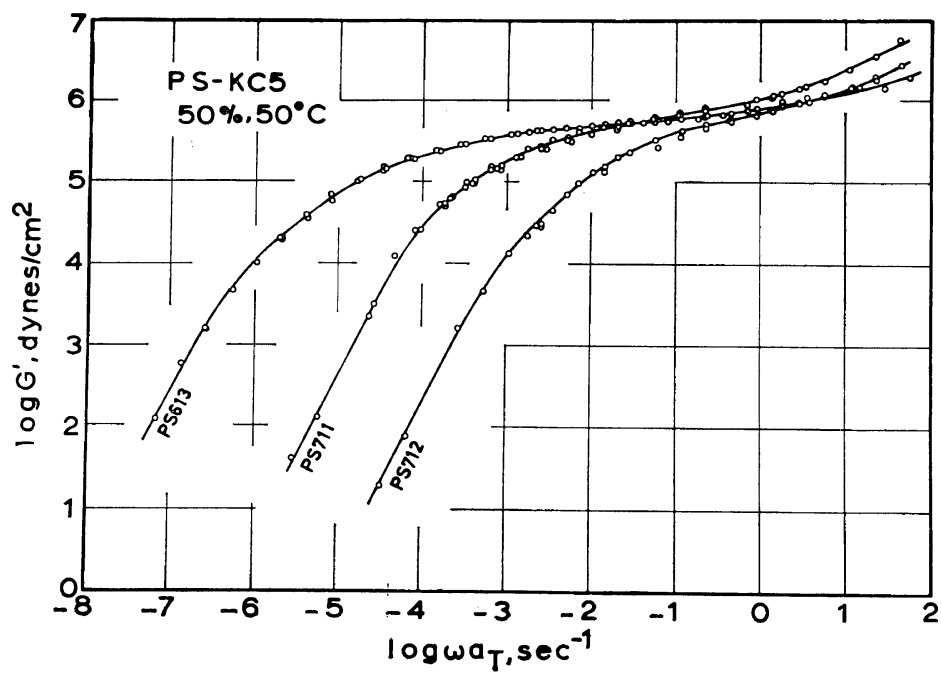

Figure 6. Master curves of $G^{\prime}$ for the $50-\mathrm{wt} \%$ solutions of PS series in $\mathrm{KC5}$. The reference temperature is $50^{\circ} \mathrm{C}$. 


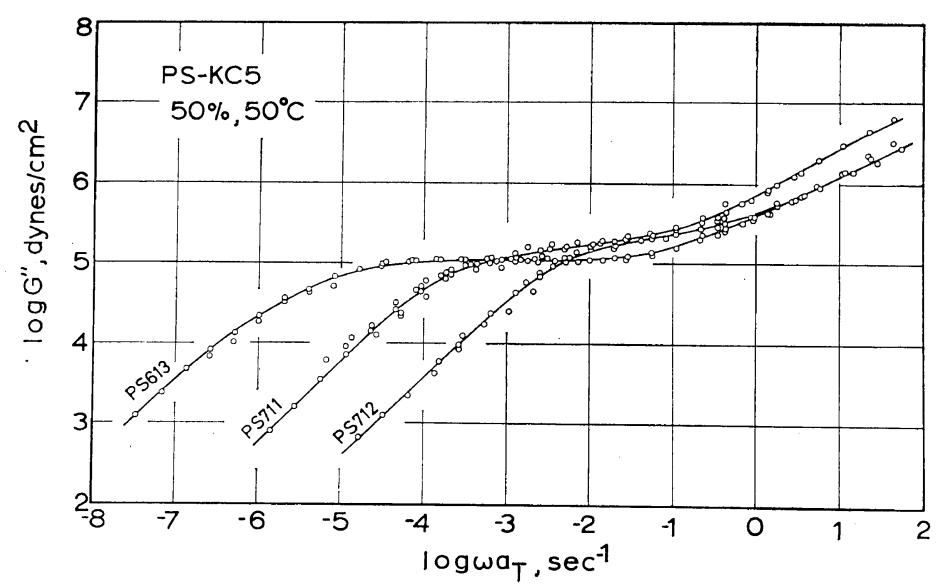

Figure 7. Master curves of $G^{\prime \prime}$ for the $50-\mathrm{wt} \%$ solutions of PS series in $\mathrm{KC5}$. The reference temperature is $50^{\circ} \mathrm{C}$.

value of $g_{\mathrm{s}}$ was calculated using the value of $M_{\mathrm{b}}$ estimated from the kinetics of copolymerization, $M_{\mathrm{b}}=9.28 \times 10^{4}$ and the Zimm-Stockmayer equation ${ }^{17}$ for randomly branched polymer:

$$
g_{\mathrm{s}}^{2}=\left[(1+\lambda M / 6)^{1 / 2}+(4 / 3 \pi) \lambda M\right]^{-1 / 2}
$$

The solid line in this figure has a slope of 0.43 , that is $g_{\eta} \propto g_{\mathrm{s}}{ }^{0.43}$. It is very interesting that this relation agrees well with that, $g_{\eta} \propto g_{\mathrm{s}}{ }^{0.4}$, expected from eq 6 and the experimental result, $[\eta]_{b} \propto M^{0.2}$.

\section{Frequency Dependences of Viscoelastic Functions}

In Figures 4 and 5 are shown the master curves of $G^{\prime}$ and $G^{\prime \prime}$ for the RB series having different molecular weights, respectively, and Figures 6 and 7 shows the similar curves for the PS series. Comparing the master curves of randomly branched polystyrenes (Figure 4) with those for linear ones (Figure 6), there is a marked difference between them. The frequency dependency curve for a branched polymer has considerable slope in the rubbery zone, where the curve for the linear one shows a rather flat plateau. Such a contrast can also be observed for the dependence curves of $G^{\prime \prime}$. It has been shown ${ }^{18,19}$ that the master curves of $G^{\prime}$ for polymers of the broad distribution of molecular weight have some slope in the rubbery plateau zone. As seen from Table III, however, the branched samples used in this study have a not so broad distribution of molecular weight, as compared with the linear
Table III. The weight and number-average molecular weights, their ratio and intrinsic viscosity at $\theta$-condition for randomly branched and linear polystyrenes.

\begin{tabular}{ccccc}
\hline Sample & $M_{w} \times 10^{-4}$ & $M_{n} \times 10^{-4}$ & $M_{w} / M_{n}$ & {$[\eta]$} \\
\hline RB10 & 610 & 480 & 1.3 & 0.556 \\
RB 9 & 220 & 140 & 1.6 & 0.428 \\
RB 8 & 130 & 74 & 1.8 & 0.412 \\
RB 7 & 49.5 & 33.0 & 1.50 & 0.335 \\
RB 6 & 30.7 & 23.1 & 1.34 & 0.290 \\
RB 5 & 20.4 & 13.6 & 1.57 & 0.258 \\
RB 4 & 12.4 & 8.58 & 1.44 & 0.219 \\
RB 3 & 7.7 & 5.33 & 1.4 & 0.185 \\
RB 2 & 6.1 & 4.20 & 1.5 & 0.156 \\
RB 1 & 3.7 & 2.26 & 1.60 & 0.115 \\
\hline PS613 & 130 & 100 & 1.3 & 0.850 \\
PS711 & 43.5 & 36.4 & 1.20 & 0.469 \\
PS712 & 20.5 & 13.2 & 1.13 & 0.328 \\
\hline
\end{tabular}

samples. Therefore, this difference in the shape of the viscoelastic functions should be due to the effect of branching. This is consistent with the result in our previous paper that the relaxation spectrum of the branched polymer is lower ${ }^{12}$ than that of linear one in the long time region. Although the height of the plateau region, $G_{\mathrm{eN}}^{0}$, cannot be evaluated, since the $G^{\prime}$ curves increase with increasing angular frequency in the region, $G_{\mathrm{eN}}^{0}$ for the randomly branched polystyrene solutions seems to be the same or 
somewhat lower than that for the linear ones having the same concentration, as seen from Figures 4 and 6 .

\section{Rheological Behavior in the Terminal Zone}

The rheological behavior of polymer liquids in the long time region or terminal zone can be characterized by such parameters as the zeroshear viscosity $\eta_{0}$, elasticity coefficient $\boldsymbol{A}_{G}$, and steady-state compliance $J_{\mathrm{e}}{ }^{0}$. These parameters are defined as

$$
\begin{aligned}
& \eta_{0}=\lim _{\omega \rightarrow 0} G^{\prime \prime} / \omega \\
& A_{G}=\lim _{\omega \rightarrow 0} G^{\prime} / \omega^{2} \\
& J_{\mathrm{e}}{ }^{0}=\lim _{\omega \rightarrow 0} J^{\prime}=A_{G} / \eta_{0}{ }^{2}
\end{aligned}
$$

In Figure $8, \eta_{0}$ for the randomly branched and linear polystyrenes used in this study are plotted logarithmically against the weight-average molecular weight. Then open circles indicate the linear polymers and the right black circles the branched ones. For linear polymer (PS

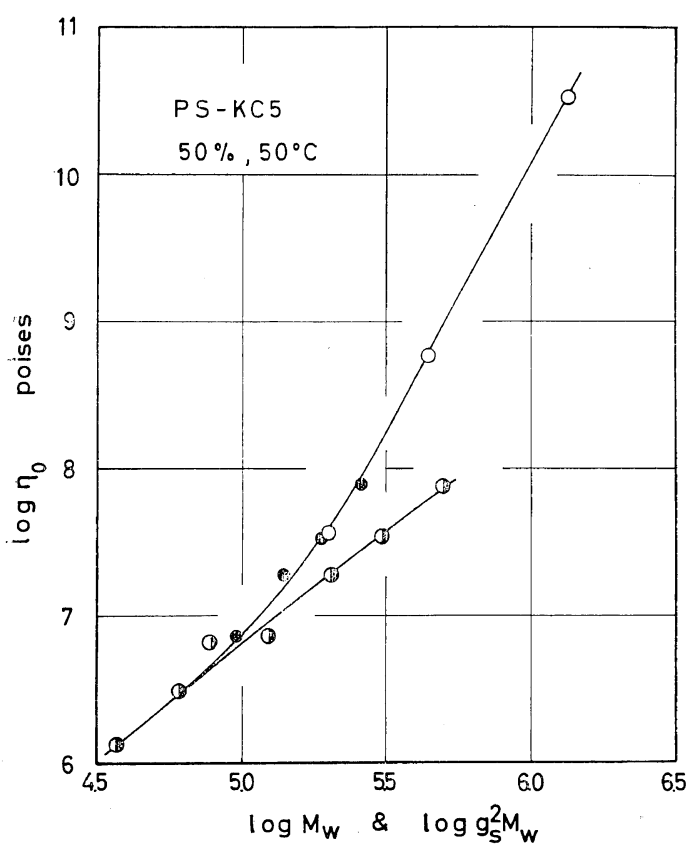

Figure 8. Molecular weight dependence of zeroshear viscosity $\eta_{0}$ for the $50-w t \%$ solutions of the RB (right black circles) and PS (open circles) series in $\mathrm{KC} 5$ at $50^{\circ} \mathrm{C}$. The black circles indicate the plots of $\log \eta_{0}$ against $\log g_{\mathrm{s}}{ }^{2} M_{w}$ for randomly branched polystyrenes. series), the molecular weight dependence of $\eta_{0}$ can be represented by a straight line having the slope of $3.5 ; \eta_{0}$ is proportional to $M_{w}^{3.5}$. $\eta_{0}$ for the RB series below the molecular weight of $1 \times 10^{5}$ smoothly join with the plot for the PS series. This shows that the lower-molecularweight polymers of the RB series should be the linear ones, and is consistent with the preceding results obtained from GPC (Figure 1), [ $\eta]$ (Figure 2) and the kinetics of copolymerization. $\eta_{0}$ for the randomly branched polymers is clearly lower than those for the linear one; $\eta_{0}$ is proportional to $M_{w}{ }^{1.5}$.

According to Bueche's theory, ${ }^{20} \eta_{0}$ of the branched polymer is determined by the dimension of the macromolecular chain and $\eta_{0}$ should be plotted against $g_{\mathrm{s}}{ }^{2} M_{w}$ instead of $M_{w}$. As seen from Figure 8 , in which $\log \eta_{0}$ is plotted against $\log g_{\mathrm{s}}^{2} M_{w}$, the relation for branched polymer coincides well with the $\log \eta_{0} v s . \log M_{w}$ curve for linear one.

In Figure 9, the values of $A_{G}$ calculated by eq 8 are plotted against the molecular weight. Also in this figure, $A_{G}$ for the RB series (right black circles) smoothly join with the line for the PS series (open circles) at lower molecular weight. As molecular weight increases, $A_{G}$ for the branched polymer deviates from and becomes lower than that for the linear one. The relation between $\log A_{G}$ and $\log M_{w}$ for RB series can be approximated by a straight line having a slope

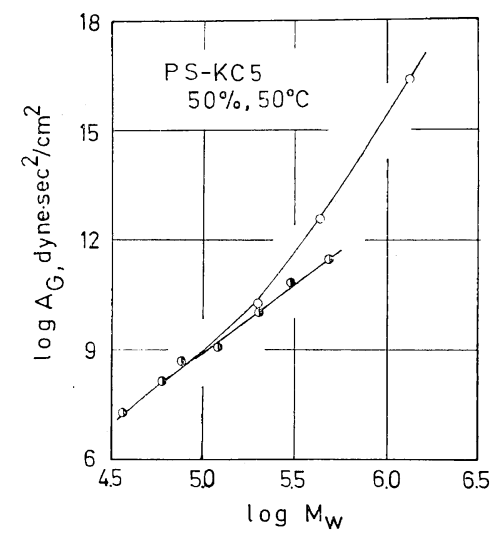

Figure 9. Molecular weight dependence of elastic coefficient $A_{G}$ for the 50-wt $\%$ solutions of the RB (right black circles) and PS (open circles) series in $\mathrm{KC} 5$ at $50^{\circ} \mathrm{C}$. 
of 3.9. This lower dependence of $\eta_{0}$ and $A_{G}$ on molecular weight for randomly branched polymers shows marked contrast with the results for the star-shaped polystyrenes ${ }^{12}$ having a constant number of branches per molecule. A similar result for the comb-shaped polystyrenes has been reported, ${ }^{11}$ when the molecular weight is increased by grafting branches of constant length on to the backbone chain.

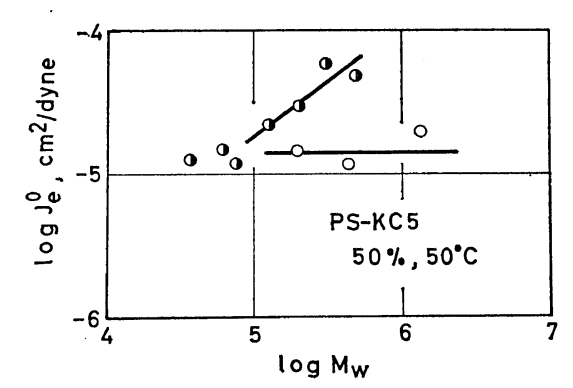

Figure 10. Molecular weight dependence of steadystate compliance $J_{e^{0}}$ for the 50 -wt $\%$ solutions of the RB (right black circles) and PS (open circles) series in $\mathrm{KC} 5$ at $50^{\circ} \mathrm{C}$.

In Figure 10 are shown the molecular weight dependences of the steady-state compliance $J_{\mathrm{e}}{ }^{0}$ calculated from eq 9 for the randomly branched and linear polystyrenes. As seen from t'is figure, $J_{\mathrm{e}}{ }^{0}$ for a linear polymer is almost independent of the molecular weight and that for the randomly branched polymer increases with the molecular weight. The straight line drawn for the branched polymer has a slope of 0.84 . Moreover, it is very interesting to note that the two straight lines seem to intersect at around $M_{w}=1 \times 10^{5}$, the average molecular weight between branch points, as was estimated before. If the behavior of a polymer liquid can be represented by a Maxwell element, the relaxation time can be given by $J_{e}^{0} \eta_{0}$. The molecular weight dependences of $\eta_{0}$ and $J_{\mathrm{e}}{ }^{0}$ shown in Figures 8 and 10 suggest that the relaxation time is proportional to $M_{w}{ }^{2.3}$ and $M_{w}^{3.5}$ for the randomly branched and linear polystyrene solutions, respectively.

\section{Concentration Dependence of Viscoelastic Func-} tions

In Figure 11, are shown the concentration dependences of $J_{\Theta}{ }^{0}$ for the linear sample, PS711, and two branched ones, RB5 and RB7. The

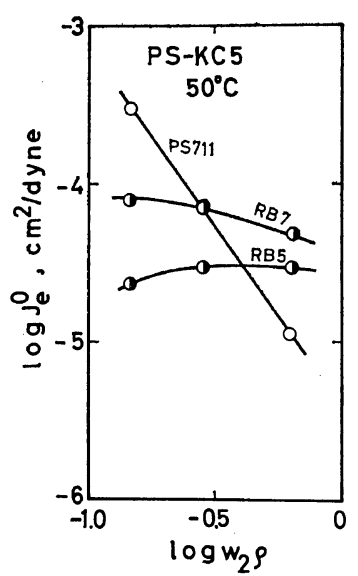

Figure 11. Concentration dependence of steadystate compliance $J_{\mathrm{e}}{ }^{0}$ for the solutions of PS711, $\mathrm{RB} 7$, and RB5 in $\mathrm{KC5}$ at $50^{\circ} \mathrm{C}$.

concentration, $w_{2} \rho$, measured as $\mathrm{g} / \mathrm{ml}$ was employed as the abscissa. This figure definitely shows how the concentration dependence of $J_{\mathrm{e}}{ }^{0}$ for randomly branched polymer differs from that for a linear one. It is well known ${ }^{21-23}$ that $J_{\theta}{ }^{0}$ for linear polystyrene is independent of molecular weight and proportional to $\left(w_{2} \rho\right)^{-2}$, so long as the molecular weight exceeds the critical molecular weight for entanglement, $M_{\mathrm{c}}$. For randomly branched polystyrene, on the other hand, the concentration dependence of $J_{\mathrm{e}}{ }^{0}$ is very low as compared with that for linear polymers. Since the frequency dependence curves, at least for the 50- and 20-wt $\%$ solutions, show a clear rubbery plateau, it has been confirmed that entanglement couplings are formed in these solutions. The higher dependence of $J_{\mathrm{e}}^{0}$ on molecular weight (Figure 11) and the lower dependence of that on concentration (Figure 12) would be distinctive features common to branched polymers. Similar results were obtained for the star-shaped polystyrene solutions as well. ${ }^{24}$

Acknowledgement. The authors wish to thank Professor M. Kurata, Institute for Chemical Research, Kyoto University, for his helpful discussions concerning the characterization of branched polymers. This study was supported in part by a grant from the Toray Science Foundation. 
Viscoelastic Properties of Concentrated Solutions of Randomly Branched Polystyrenes

\section{REFERENCES}

1. A. Charlesby, J. Polym. Sci., 17, 379 (1955).

2. L. D. Moore, Jr., ibid., 36, 155 (1959).

3. L. H. Tung, ibid., 46, 409 (1960).

4. W. H. Peticolas, ibid., 58, 1405 (1962).

5. V. C. Long, G. C. Berry, and L. M. Hobbs, Polymer, 5, 517 (1964).

6. S. Onogi, S. Kimura, T. Kato, T. Masuda, and N. Miyanaga, J. Polym. Sci., Part C, 15, 381 (1966).

7. W. W. Graessley and J. S. Prentice, ibid., Part $A-2,6,1887$ (1968).

8. G. Kraus and J. T. Gruber, ibid., Part A, 3, 681 (1965).

9. D. P. Wyman, L. Elyash, and W. J. Frazer, ibid., Part A, 3, 681 (1965).

10. J. E. Guillet, R. L. Combs, D. F. Slonaker, D. A. Weemes, and H. W. Coover, Jr., J. Appl. Polym. Sci., 9, 767 (1965).

11. T. Fujimoto, T. Narukawa, and M. Nagasawa, Macromolecules, 3, 57 (1970).

12. T. Masuda, Y. Ohta, and S. Onogi, Macromolecules, 4, No. 6 (1971), to be published.
13. H. Markovitz, J. Appl. Phys., 23, 1070 (1952).

14. P. J. Flory, "Principles of Polymer Chemistry," Conell University, Press, Ithaca, N. Y., 1953.

15. L. J. Young, "Polymer Handbook," J. Brandrup and E. H. Immergut Ed., Interscience Publishers, New York, N. Y., 1965, p II-291.

16. C. D. Thurmond and B. H. Zimm, J. Polym. Sci., 8, 477 (1952).

17. B. H. Zimm and W. H. Stockmayer, J. Chem. Phys., 17, 1301 (1949).

18. T. Masuda, K. Kitagawa, T. Inoue, and S. Onogi, Macromolecules, 3, 116 (1970).

19. S. Onogi, T. Masuda, and K. Kitagawa, Macromolecules, 3, 109 (1970).

20. F. Bueche, J. Chem. Phys., 40, 484 (1964).

21. T. Kotaka and K. Osaki, J. Polym. Sci., Part $C, 15,453$ (1966).

22. W. W. Graessley and L. Segal, Macromolecules, 2, 49 (1969).

23. D. C. Bogue, T. Masuda, Y. Einaga, and S. Onogi, Polymer J., 1, 563 (1970).

24. Y. Ohta, T. Masuda, M. Kitamura, and S. Onogi, in preparation. 